\title{
Comparison of applicable adjustment procedures for very small batches
}

\author{
Daniel Duret and Alain Sergent* \\ SYMME Laboratory, University of Savoie, BP 80439, 74944 Annecy-le-Vieux Cedex, France
}

Received: 29 April 2017 / Accepted: 16 March 2019

\begin{abstract}
The objective of this study is to propose and compare three methods of production process control that are voluntarily simple to implement on a machine. A mathematical formulation of the "sequence" type is used to model each method. An experimental validation is then carried out on the principle of launching a preproduction consisting of five parts machined and measured consecutively. A graphical analysis makes it possible to compare the different methods proposed and makes it possible to show the interest of the Bayesian weighted adjustment method.
\end{abstract}

Keywords: Adjustment method / Bayesian weighted adjustment / process control / mass production

\section{Introduction}

In mass production, the chosen adjustment method is based on the laws of sampling. This requires making parts before making a decision [1,2]. When parts are expensive or for launching small series, it may be advantageous to react quickly on the adjustment to converge toward satisfactory production [3]. Some authors propose to work from similar processes to obtain more relevant information [4]. In this article, we propose to compare three methods of adjustment, simple to use, intended to be used directly in the workshop, and if possible quickly converging to compliant parts. The three procedures are based respectively on the following methods:

- A part-to-part correction standard method, the corrections applied to the part $(\mathrm{i}+1)$ are directly derived from the information taken from the part (i);

- A method of correction weighted according to the rank of production (progressive reconstitution of a sample to get closer to the spirit of Shewhart's maps);

- A weighted correction method like the previous one, based on a Bayesian approach of the adjustment, that is to say, being aware that the setting retains a random appearance, therefore not stable.

We propose to test these three methods by machining from a series of pieces on a CNC machining center (three axis). The corrections made will be calculated from the previous measurements carried out on a CMM (digital three-dimensional measuring machine) according to the

\footnotetext{
* Corresponding author: sergent@univ-smb.fr
}

three proposed adjustment methods. For the comparison to be valid, we will start from the same initial setting conditions. In the end, the weighted correction methods seem efficient and in particular the Bayesian method seems well adapted for a poorly controlled means of production.

\section{Sampling adjustment problem}

Production is considered as a random process, variable $X$ depending on an adjustment parameter $\theta$. $\theta$ is the difference between the mean of the production $\mu$ (unknown) and the target value. $\theta$ is a parameter of adjustment that will be sought to tend toward zero as quickly as possible Figure 1 (gains of time and means of production) [5].

$\theta$ is unknown, the rest of the study will concern its estimation and spread in order to correct the adjustment of the means of production.

\section{Typical correction from a sample}

If we take a sample of $n$ individual values for the variables $X_{1}$ (first part of the sample) $\ldots X_{i}, \ldots X_{n}$ (last piece of the sample), considered a priori as totally independent ( $\theta$ supposed fixed for the complete sample), the control of the adjustment most often amounts to analyze the event $A$, a posteriori equation (1):

$$
A=\{X=\bar{x}\}
$$

This method allows to propose a correction $\delta$ (assumed to cancel $\theta$ thank to the estimate of $\mu$ by $\bar{x}$ ) equation (2). 


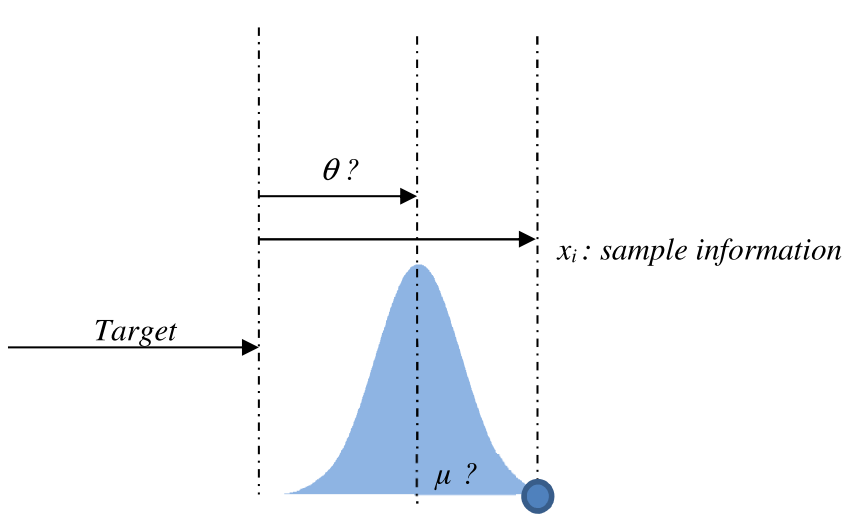

Fig. 1. Example of graphical presentation of the law of the variable $X$.

Recall that since $\mu$ is unknown, we hope that $\bar{x}$ gives a good estimate.

$$
\text { Either } \delta=-\bar{x} \text {. }
$$

The sample, by its mean $\bar{x}$, gives an estimate of $\theta$. With the hypothesis of a Gaussian distribution, one can have an idea of the associated confidence interval equation (3):

$$
\operatorname{Pr}\left(\bar{x}-z_{1-\alpha / 2} \frac{\sigma}{\sqrt{n}} \leq \theta \leq \bar{x}+z_{1-\alpha / 2} \frac{\sigma}{\sqrt{n}}\right)=1-\alpha .
$$

This approach is better suited to the mass production, and this for parts not too expensive [1]. The size of the sample will depend on the cost and time of production of a part.

\section{Part-to-part correction: method 1}

The idea is not to have for a complete sample (of $n$ parts) before deciding on a correction. Each piece will give chronological information (comparison of the measured value $x_{i}$ with the target value) making it possible to define instantly a correction equation (4):

$$
\delta_{1}=-x_{1} .
$$

To the first part, then equation (5):

$$
\delta_{k}=-x_{k} .
$$

To the $k$ th part.

The risk with this approach is perhaps to propose a method of adjustment too brutal, more likely to generate an adjustment oscillation around the target.

In the following, we will propose and experimentally compare two new methods of adjustment part to part. The two methods described below having as objective, to lead to a convergence of the adjustment more stable and especially faster, and therefore less costly.

\section{Weighted piece-to-part correction: method 2}

The first part is considered a sample of one part. We corrected, as for the first method, to equation (6):

$$
\delta_{1}=-x_{1} \text {. }
$$

After this first adjustment, correction has been made, we obtain a second part of dimension $x_{2}$.

At this step, we have two pieces of information $\left(x_{1}\right.$ and $x_{2}$ ) as for a sample of two parts (part 1 and part 2). In fact, these two values are not independent. This is why we will consider that if we had not made the first adjustment, we would have obtained as value for the second part equation (7):

$$
x_{2}^{*}=x_{2}-\delta_{1}=x_{2}+x_{1} .
$$

If we had waited for two pieces (conventional sampling) and to correct the average, this would give equation (8):

$$
\delta_{2}^{*}=-\frac{x_{1}+x_{2}^{*}}{2}=-x_{1}-\frac{x_{2}}{2}=\delta_{1}-\frac{x_{2}}{2} .
$$

However, since the adjustment has already been partially after obtaining the first part, after obtaining this second part, we only correct of equation (9):

$$
\delta_{2}=-\frac{x_{2}}{2} .
$$

After these two adjustments, we obtain a third part of dimension $x_{3}$. By applying the same reasoning, one can imagine that another average value would have been obtained if these adjustments had not been made immediately equation (10):

$$
x_{3}^{*}=x_{3}-\delta_{1}-\delta_{2}=x_{3}+x_{1}+\frac{x_{2}}{2} .
$$

If we had waited to have three parts and correct the average, this would give equation (11):

$$
\begin{aligned}
\delta_{3}^{*} & =-\frac{x_{1}+x_{2}^{*}+x_{3}^{*}}{3} \\
& =-\frac{x_{1}+\left(x_{2}+x_{1}\right)+\left(x_{3}+x_{1}+x_{2} / 2\right)}{3} \\
& =\delta_{1}+\delta_{2}-\frac{x_{3}}{3}
\end{aligned}
$$

The first two adjustments have already been made, with the information obtained in the third part, we correct of equation (12):

$$
\delta_{3}=-\frac{x_{3}}{3}
$$

At the $k$ th piece we will correct of equation (13):

$$
\delta_{k}=-\frac{x_{k}}{k}
$$

If one has an estimate of the setting noise $(\sigma(\theta))$ characterizing the lack of control of the latter, this will stop this procedure and will admit that the adjustment is optimized equation (14).

For example:

$$
\left|\delta_{k}\right| \leq 2 . \sigma(\theta) .
$$




\section{Weighted part-to-part correction using a Bayesian approach: method 3}

In the previous approaches, we assumed that the setting $\theta$ was unknown but fixed. With a Bayesian approach, $\theta$ is considered as a random variable (unstable setting over time). This implies that, following a given adjustment action (due to the choices of operators, tools, etc.), we have in fact a real adjustment that oscillates around an average value with a dispersion characterized by $\sigma(\theta)$ equation (15). $\sigma_{p}$ represents the standard deviation of the intrinsic production dispersion.

$$
\delta=-\theta_{M}=-\frac{1}{1+\frac{\sigma_{p}^{2}}{k \cdot \sigma^{2}(\theta)}} \bullet \bar{x} .
$$

The dispersion of adjustment $\sigma(\theta)$ is rather difficult to estimate. In recent work, we obtained experimentally a standard deviation $\sigma(\theta)$ of substantially the same order as the instantaneous standard deviation of production $\sigma_{p}$, Tables 1 and 2 .

A pocket will be machined on small lots of 5 parts. The 5 parts are machined without changing settings. The lots will be independent, for each time a new production starts (sequential manufacture of small batches) with measurement and entry of new setting parameters. Each part will be measured two-dimensional position of $X$ and $Y$.

The diameter $D$ is independent of the setting position of the workpiece, but depends on the programming (circular path of the cutter center) and the radius of the cutter. By cons, location dimensions are highly dependent on the use of clamping means (vise with fixed stop).

The proposed theoretical correction $\left(\delta=-\theta_{M}\right)$ at the maximum likelihood is given by equation (16):

$$
\delta=-\theta_{M} \approx-\frac{1}{1+\frac{1}{k}} \bullet \bar{x} .
$$

Let us go back to the previous procedure, using this formula.

The first piece is considered a sample as of a part. We are correcting with equation (17):

$$
\delta_{1}=-\frac{1}{1+\frac{1}{1}} x_{1}=-\frac{1}{2} x_{1}
$$

After this first adjustment we obtain a second part with a value $x_{2}$.

We will consider that if we had not made the first adjustment, we would have obtained as value for the second part equation (18):

$$
x_{2}^{*}=x_{2}-\delta_{1}=x_{2}+\frac{1}{2} x_{1} .
$$

If we had waited to have two parts and correct of the average, it would give equation (19):

$$
\begin{aligned}
\delta_{2}^{*} & =-\frac{1}{1+\frac{1}{2}} \bullet \frac{x_{1}+x_{2}^{*}}{2}=-\frac{x_{1}+x_{2}+\frac{1}{2} x_{1}}{3} \\
& =-\frac{1}{2} x_{1}-\frac{x_{2}}{3}=\delta_{1}-\frac{x_{2}}{3} .
\end{aligned}
$$

Table 1. Short time dispersions.

\begin{tabular}{llll}
\hline Parameter & $X$ & $Y$ & $D$ \\
\hline$\sigma$ Measure noise $(\mathrm{mm})$ & 0.002 & 0.002 & 0.004 \\
$\sigma$ Short-time $(\mathrm{mm})$ & 0.345 & 0.224 & 0.021 \\
\hline
\end{tabular}

Table 2. Dispersion of setting.

\begin{tabular}{llll}
\hline Parameter & $X$ & $Y$ & $D$ \\
\hline$\mu_{\theta}$ Setting $(\mathrm{mm})$ & -0.035 & 0.018 & 0.076 \\
$\sigma_{\theta}$ Setting $(\mathrm{mm})$ & 0.309 & 0.185 & 0.027 \\
\hline
\end{tabular}

Thus, since the adjustment has already been partially made after the first part by $\delta_{1}$, after obtaining this second part, we correct equation (20):

$$
\delta_{2}=-\frac{x_{2}}{3}
$$

After these two adjustments, we obtain a third part of dimension $x_{3}$. By applying the same reasoning, we can imagine that another average value would have been obtained if these adjustments had not been made immediately equation (21):

$$
x_{3}^{*}=x_{3}-\delta_{1}-\delta_{2}=x_{3}+\frac{1}{2} x_{1}+\frac{1}{3} x_{2} .
$$

If we had waited to have three parts and correct to average, this would give equation (22):

$$
\begin{aligned}
\delta_{3}^{*} & =-\frac{1}{1+\frac{1}{3}} \frac{x_{1}+x_{2}^{*}+x_{3}^{*}}{3} \\
& =-\frac{1}{4}\left(x_{1}+\left(x_{2}+\frac{1}{2} x_{1}\right)+\left(x_{3}+\frac{1}{2} x_{1}+\frac{1}{3} x_{2}\right)\right) \\
\delta_{3}^{*} & =\delta_{1}+\delta_{2}-\frac{x_{3}}{4}
\end{aligned}
$$

The first two adjustments have already been made, after the information obtained with the third part, we correct of equation (23):

$$
\delta_{3}=-\frac{x_{3}}{4}
$$

After the $k$ th piece we will correct of equation (24):

$$
\delta_{k}=-\frac{x_{k}}{k+1}
$$




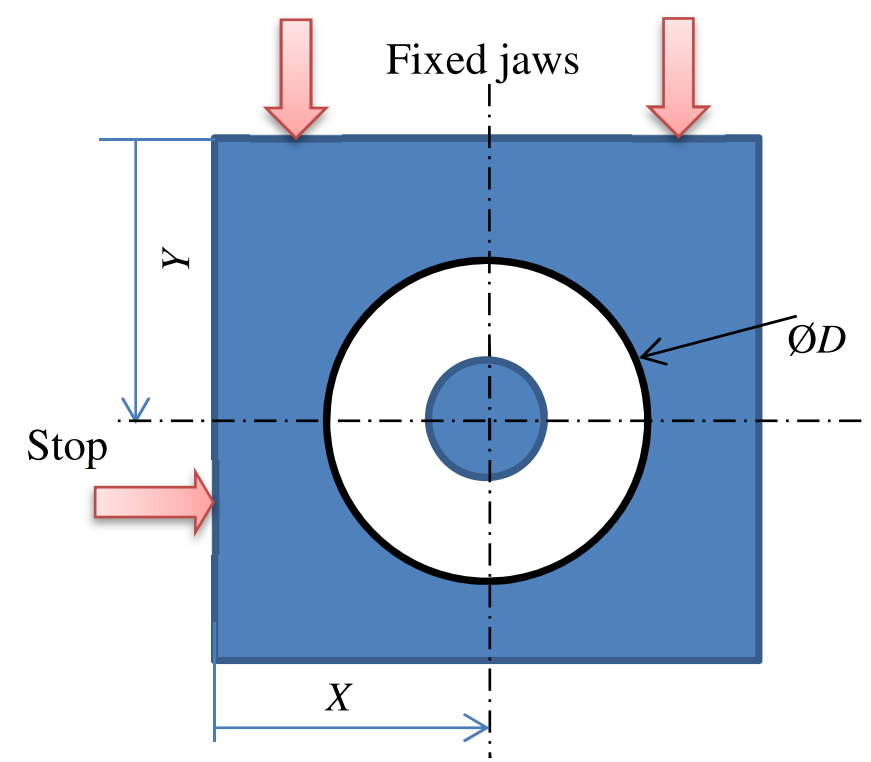

Fig. 2. Production of a circular groove (seen from above).

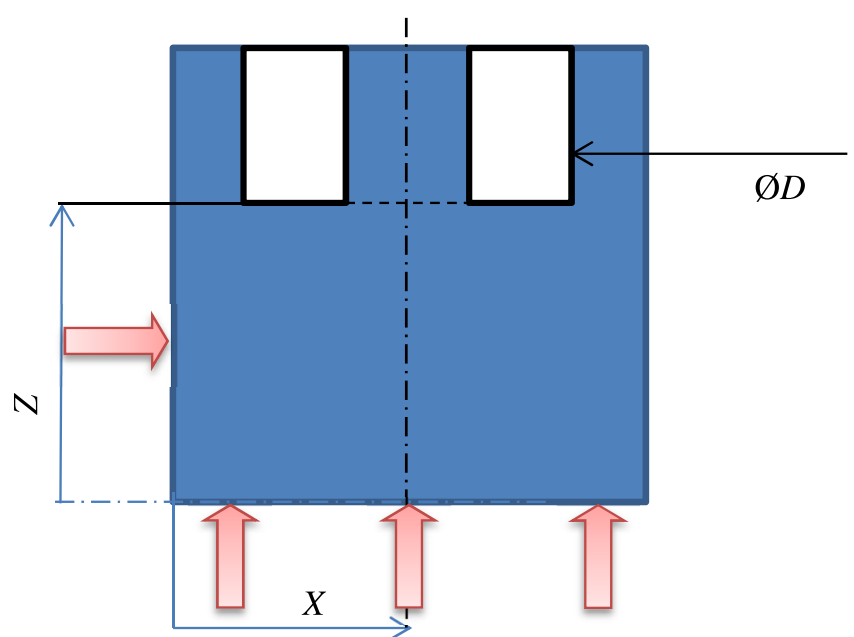

Fig. 3. Parameter $Z$ of the height of the circular groove (front view in section).

\section{Experimental study}

\subsection{Machining a circular groove}

\subsubsection{Setting and positioning}

The diameter $D$ is independent of the positioning of the part, but depends on the programming (circular path of the center of the cutter) and the radius of the cutter Figures 2 and 3. On the other hand, the location coordinates are highly dependent on the grip of the workpiece (vice with fixed stop).

\subsubsection{Characterization of the means of production}

The production means chosen for the study is a FanucRobodrill $\alpha$-T14 i Fb CNC machining center Figure 4, equipped with a Fanuc digital interface control. These

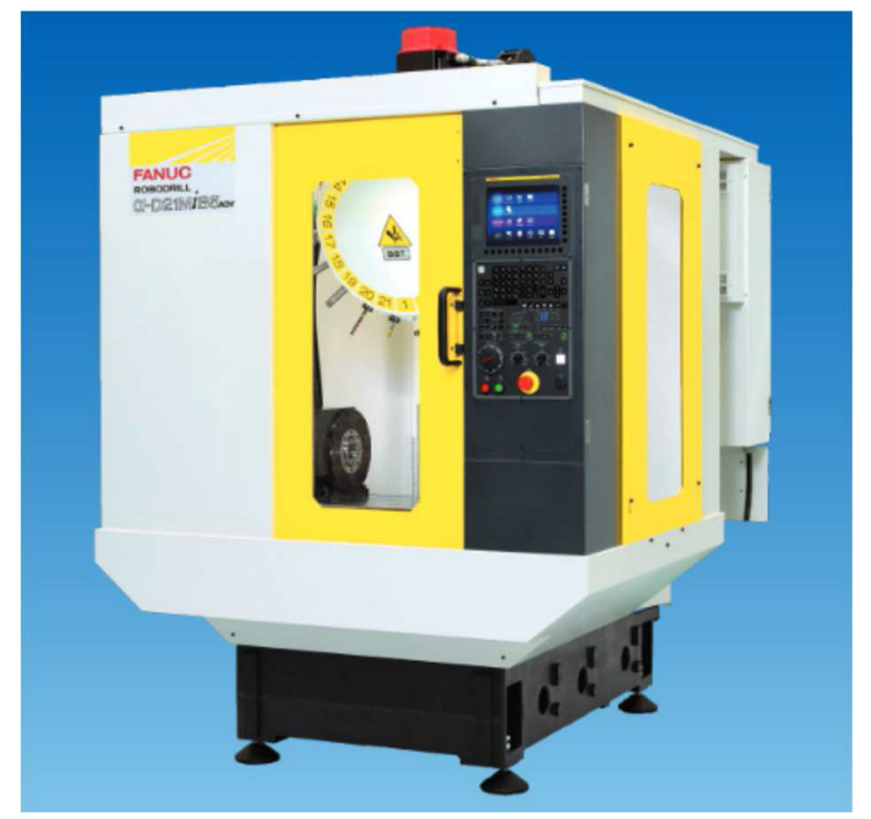

Fig. 4. Means of production for the machining of parts.

Table 3. Experimental estimation of the intrinsic dispersions of the process.

\begin{tabular}{lllll}
\hline Parameters & $X$ & $Y$ & $D$ & $Z$ \\
\hline$\sigma$ Short term $(\mathrm{mm})$ & 0.345 & 0.224 & 0.020 & 0.157 \\
\hline
\end{tabular}

equipments correspond to production means prevalent in small and medium series conventional mechanics. This means is renowned for its rigidity and mechanical robustness.

For this classical means of production, we were able to estimate the intrinsic dispersions of the process on the principle of a previous experimental study where 29 samples of 5 parts were made [2]. All these samples were obtained by following the same procedure, without correction during the complete machining of the sample, which makes it possible to estimate the intrinsic dispersions of the process [6,7], Table 3 .

We note that the means of production disperse much, particularly the support on the point stop (X axis), the stop being much less rigid than the fixed jaw of the vice (Y axis).

For this experimental study on the adjustment (comparison of three methods), carried out on the same machine, with the same operators over a short period, the results should be better. The first part of each tested method was made with exactly the same setup instructions (program origin, gauge, program). If we consider that we have a sample of three parts (results for the first part of each method), this gives the following results Table 4, estimating the standard deviation by the range of the range small sample, estimated with $d_{2}{ }^{*}$ ) [8]. 


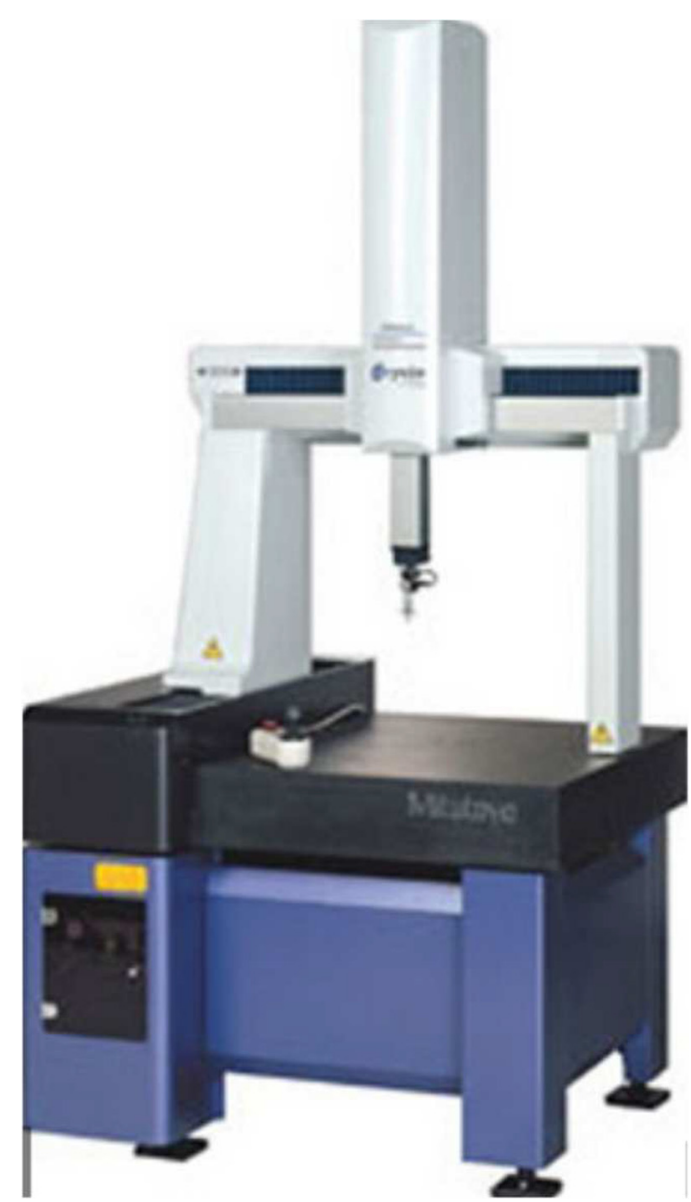

Fig. 5. Digital three-dimensional measuring machine.

Table 4. Estimation of the short term dispersion of the process control.

\begin{tabular}{lllll}
\hline Parameters & $X$ & $Y$ & $D$ & $Z$ \\
\hline Target value & 25.000 & 25.000 & 35.000 & 44.000 \\
$\sigma$ Short term $(\mathrm{mm})$ & 0.032 & 0.038 & 0.025 & 0.020 \\
\hline
\end{tabular}

Table 5. Estimation of the measurement dispersion of the control means.

\begin{tabular}{lllll}
\hline Parameters & $X$ & $Y$ & $D$ & $Z$ \\
\hline $\begin{array}{l}\sigma \text { Measurement } \\
\text { noise (mm) }\end{array}$ & 0.002 & 0.002 & 0.004 & 0.005 \\
\hline
\end{tabular}

\subsubsection{Means of measurement}

The measurements were carried out on a three-dimensional Mitutoyo Crysta 544 measuring machine Figure 5. The manufacturer indicates a maximum measurement deviation of: $(1.9+0.3 \mathrm{~L} / 100) \mu \mathrm{m}$. This machine integrate a thermal compensation.
Table 6 Control of the position $(X ; Y)$ of the circular groove.

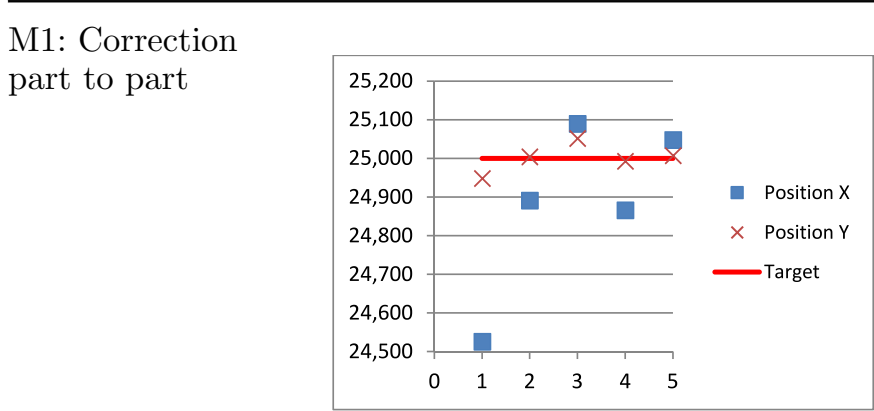

M2: Weighted correction

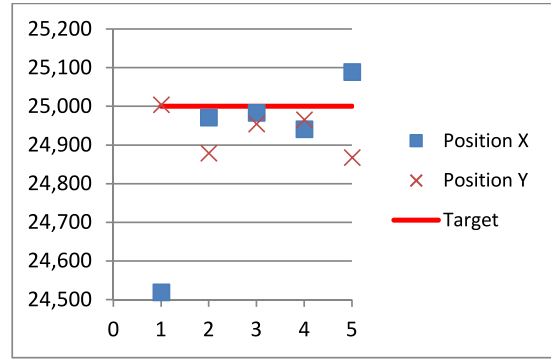

M3: Bayesian weighted correction

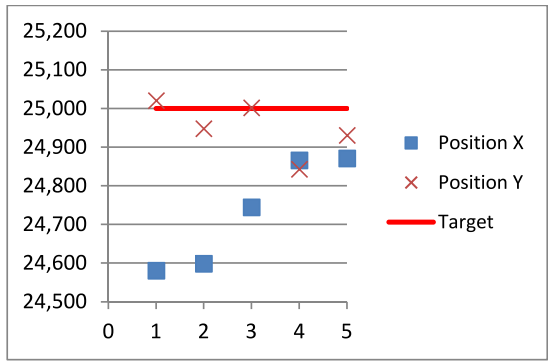

The estimation of the dispersions of the measurement process was obtained by measuring about 20 times the same part. This series of measurements indicates Table 5 .

As a result of this comparison of methods, the measurement noise will be neglected.

\subsection{Comparison of three methods}

Based on the same set-up instructions, we applied the three procedures described above to 5 parts. If the adjustment was perfectly controlled and the production did not disperse, we should have the same first value for part number 1 .

\subsubsection{Position of the circular groove}

The figures given in Table 6 show the convergence of the process with respect to the target for the three methods of 
Table 7 Control of the depth $(Z)$ of the circular groove.

M1: Correction part to part

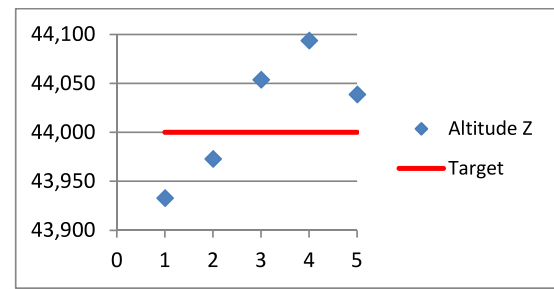

M2: Weighted correction

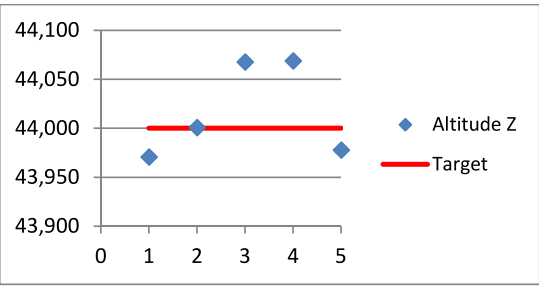

M3: Bayesian weighted correction

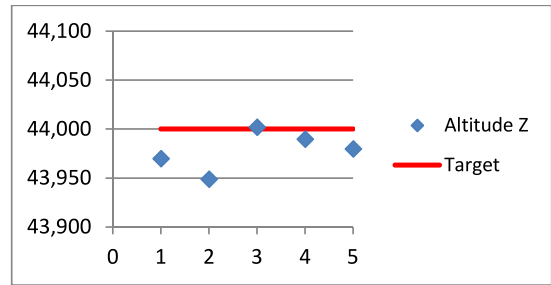

controlling the position of the circular groove tested. In this case, the control of the position (X, Y) of the hole depends on 2 factors of equal importance:

- Corrections obtained by the method used (1, 2 or 3$)$;

- Errors in restoring the workpiece.

In this situation, the three methods give simulated results: an effective correction at the start of machining then an unstable oscillation around the targeted target because of the setting in variable position.

\subsubsection{Altitude of the bottom of the circular groove}

The figures in Table 7 compare the three methods of piloting the bottom of the circular groove with respect to the target. The machining height is a parameter or the positioning is more stable (plane support). The efficiency of method 3 is clear. Convergence and stability of the adjustment around the target is good.

\subsubsection{Diameter of the circular groove}

The figures in Table 8 compare the three methods of controlling the diameter of the circular groove with respect to the target. This parameter is entirely controlled by the program (insensitive to human
Table 8 Piloting the diameter of the circular groove.

\section{M1: Correction}

part to part

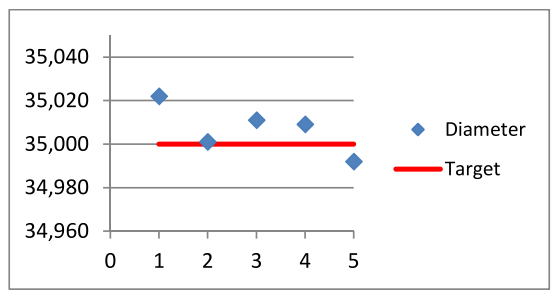

M2: Weighted

correction

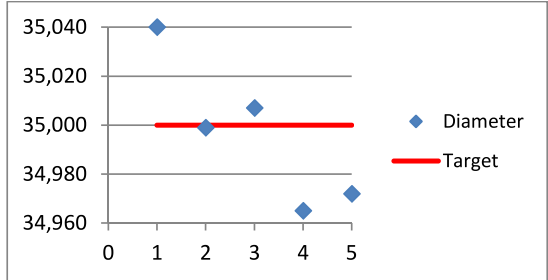

M3: Bayesian

weighted

correction

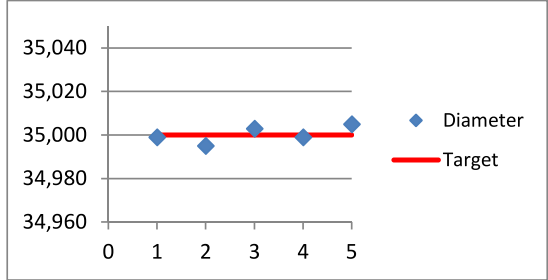

influence). In this case, method 3 is even more efficient and provides stability around the target more efficient than methods 2 and 3.

\section{Conclusions}

The objective of this study was to compare simple methods for adjusting the means of production, using an algorithm that is not very complex and can be applied directly to the process in real-time. Further experiments would confirm the promising results obtained by the adjustment methods 2 and 3 .

Indeed, we have already shown that the weighted methods 2 and 3 avoid the "pumping" of the direct method 1. They converge toward the target faster. These methods should be particularly attractive for all parts with high added value or expensive raw material, such as the luxury watch industry, the aerospace industry and so on. This should make it possible to avoid the production of parts outside tolerance more quickly.

In particular, the Bayesian weighted method seems well adapted when, for a given setting (setpoint), the actual setting on the means of production is poorly controlled and disperses a lot (e.g., strong influences, operators, positioning, measurement of tools, etc.). 


\section{References}

1. W.A. Shewhart, Economic control of quality of manufactured products (Van Nostrand, New York, MacMillan, London, 1931) (1980 by American Society for Quality)

2. BSI British Standards BSI 5702-3:2008, Guide to statistical process control (SPC) charts for variables - Part 3: Charting techniques for short runs and small mixed batches (2008)

3. M. Pillet, A specific SPC chart for small-batch control, Qual. Eng. 8, 581-586 (1996)

4. M. Wiederhold, J. Greipel, R. Ottone, R. Schmitt, Clustering of similar processes for the application of statistical process control in small batch and job production, Int. J. Metrol. Qual. Eng. 7, 404 (2016)

5. D. Duret, M. Pillet, Z. Zhou, Process adjustment using a Bayesian approach, Cogent Eng. 2, (2015)

6. M.H. Bui, F. Villeneuve, A. Sergent, Evaluation of different methods in quantification of manufacturing defects, Asian Int. J. Sci. Technol. Prod. Manuf. Eng. 4, (2011)

7. S. Tichadou, O. Legoff, J.-Y. Hascoet, 3D manufacturing dispersions: two experimental applications, in: CIRP (2007)

8. D. Duret, Qualité de la mesure en production (EYROLLES, Paris, 2008)

Cite this article as: Daniel Duret, Alain Sergent, Comparison of applicable adjustment procedures for very small batches, Int. J.

Metrol. Qual. Eng. 10, 6 (2019) 